\title{
Spontaneous Formation of Graphene on Diamond (111) Driven by B-doping Induced
}

\section{Surface Reconstruction}

Chao $\mathrm{Lu}^{1,2}$, Hongxin $\mathrm{Yang}^{3}$, Jing $\mathrm{Xu}^{4}$, Lifang $\mathrm{Xu}^{1}$, Mairbek $\mathrm{Chshiev}^{3}$, Shengbai Zhang ${ }^{5}$ and Changzhi $\mathrm{Gu}^{1,6,7 *}$

\begin{abstract}
Spontaneous construction of graphene on boron-doped diamond (111) surface has been found in first-principles calculations. Boron-doping-induced surface reconstruction is the mechanism responsible for the diamond-to-graphene phase transition. Furthermore, the effect on the surface is unexpectedly observed at any doping depth down to the 7th layer, and a low concentration of substituent boron - only $1 / 4-$ is effective for graphene formation. Amazingly, when boron atoms are incorporated into the fifth layer, the direct optimization of the $1 \times 1$ surface automatically gives rise to a graphene structure from the first bilayer with no energy barrier, indicating that the formation of graphene is spontaneous. These findings provide an alternative strategy for graphene synthesis on wide-gap insulators with high thermal conductivity.
\end{abstract}




\section{Introduction}

Since successfully "re-discovery" in 2004 [1, 2], graphene has been a material of great interest owing to its very good thermal conductivity, mechanical stiffness and extraordinary electronic transport properties [3-5], including high carrier mobility and ballistic transport, which are generally preserved up to room temperature [6-8]. These properties give graphene great potential as a material for technological applications as a successor of silicon in the post-Moore's law era [9-11], in spintronics [12-15] and in quantum computing [16]. One of the main requirements for such applications is ability to grow uniform graphene on an insulating substrate [17]. Unlike suspended graphene, which is a semi-metal $[1,18]$, epitaxially grown graphene on an insulator may open a bandgap at the Dirac point due to the breaking of sublattice symmetry induced by the graphene-substrate interaction $[19,20]$. Two insulating materials that have been used very successfully as a substrate for graphene growth/synthesis are $\mathrm{SiC}$ and hexagonal boron nitride (hBN) [21-23]. Recently, graphene-on-diamond structures and devices showed greatly improved performance, due to the excellent properties of diamond substrate [24-28]. However, few investigation is reported so far regarding the possibility of synthesizing graphene directly on diamond [29]. Moreover, a very small lattice mismatch between graphene and the diamond (111) surface makes the latter an ideal candidate as a substrate for graphene. Investigating the possibility of graphene growth on a diamond substrate is the goal of the present work, which may provide a viable alternative for graphene synthesis on C-terminated $\mathrm{SiC}(0001)$ and $\mathrm{hBN}[30,31]$, and of earlier reports of local graphitization on diamond (111) surface [32, 33]. This method of graphene synthesis on diamond is an alternative for overcoming the overheating problem in graphene-based devices, in view of the high thermal conductivity of diamond combined with the huge electrical conductivity of graphene.

In this letter, we report systematically investigating the possibility of graphene construction on boron doped diamond (111) surface for various doping concentrations and depths, using first-principles calculations, finding that graphene can form spontaneously without any potential barrier when B is incorporated in surface layers with a relatively low concentration. In contrast to what might be expected, the doping's effect on the surface is stronger as the B atoms are located deeper, until the 7th layer, where the situation is like substitution in bulk. Boron-doping induced surface reconstruction is considered to be the physical mechanism of the diamond-to-graphene phase transition.

\section{Methods}


Our first-principles calculations were carried out using the Vienna ab initio simulation package (VASP) [34]. The electron-core interactions were described by the Vanderbilt ultrasoft pseudopotential [35] and the exchange correlation energy obtained within the generalized gradient approximation (GGA) [36]. A plane wave basis set was used to expand the Kohn-Sham orbitals, with a kinetic energy cutoff of $350 \mathrm{eV}$. The theoretical lattice constant of diamond was taken to be equal to $3.574 \AA$ in all calculations, and the Monkhorst-Pack scheme [37] was used for the Brillouin zone integration with a $2 \times 2 \times 1 \mathrm{k}$-point mesh. During energy minimization, the atoms are set to be fully relaxed except those in the bottom two layers, which are fixed at their respective positions. To avoid interaction between the top and bottom surfaces, 18 layers of carbon atoms in the supercell, with the bottom layer terminated by hydrogen and more than $15 \AA$ of vacuum, was used to simulate diamond (111). The side and top views of diamond (111) are shown in Figs. 1(a) and (b), respectively. The distances for the first several bilayers (BL) are also provided, showing that the first layer of carbon atoms shifts inward and forms stronger bonds with the second layer of carbon after relaxation in pure diamond (111) surface. At the same time, the first bilayer is shifted outward and forms weak bonds with the second bilayer. However, such small change is not yet enough to separate the first bilayer of carbon atoms into graphene from the diamond phase.

\section{Results and discussions}

In order to stimulate the diamond-to-graphene phase transition, we introduced boron into diamond (111) taking into account that it represents the most common impurity in both natural and chemical vapor deposited (CVD) diamond. The stable doping position of boron in diamond is substitutional rather than interstitial [38]. Figures 2(a) and (b) show the optimized structures with boron atoms incorporated into the first and the third layers of diamond (111), respectively. One can see that with boron atoms in the first layer [Fig. 2(a)], the vertical distance between a surface B atom and a neighboring $\mathrm{C}$ atom is slightly smaller $(0.23 \AA)$ than the vertical distance between a surface $\mathrm{C}$ atom and a neighboring $\mathrm{C}$ atom in pure diamond $(0.27 \AA)$ [Fig. 1(a)]. When boron atoms are incorporated into the third layer [Fig. 2(b)], the vertical distance between the carbon atom in the first layer and the second-layer neighbor that sits on top of the boron atom is reduced from $0.27 \AA$ to $0.05 \AA$, while the distance between the first bilayer and the second bilayer is increased to $2.0 \AA$. When boron is doped into the third layer, it forms covalent bonds with the three nearest carbon atoms in the fourth layer since it has only three electrons in the outer shell. Thus, it leads to a weaker bonding with a top carbon atom 
in the second layer (denoted by $\mathrm{C}_{\text {top }}$ ), and then the interaction between the first and second bilayer becomes weaker. In this case, the $\mathrm{C}_{\text {top }}$ atom forms $\mathrm{sp}^{2}$-like hybridized bonds with the neighboring carbon atoms in the first layer, while the remaining carbon atoms in the second layer still form sp ${ }^{3}$-like bonds with their counterparts in the first layer. Additionally, one can see that $1 / 4$ boron concentration in the third layer results in 3/4 of the carbon atoms in the first layer forming $\mathrm{sp}^{2}$ bonds with $\mathrm{C}_{\mathrm{top}}$ atoms in the second layer, eliminating $3 / 4$ of the dangling bonds of the (111) surface.

Amazingly, when boron atoms are incorporated into the fifth layer, the direct optimization of $1 \times 1$ surface automatically gives rise to a graphene structure from the first bilayer with no energy barrier, indicating that the formation of graphene is spontaneous [Fig. 2(c,d)]. Being adjusted to the diamond lattice, the distance between neighbor carbon atoms within the graphene layer is about $1.45 \AA$, which is very close to the nearest-neighbor distance of $1.42 \AA$ in graphene. The distance between graphene layer and diamond substrate stabilizes at about $3.30 \AA$, which is comparable to the distance of $3.35 \AA$ between the layers in graphite. Thus, a structural phase transition from diamond to graphene on diamond (111) substrate truly occurred in this simulation. Note that the structure below the graphene layer in Fig. 2(d) is very similar to the third-layer-doping structure in Fig. 2(b). The same phase transition happens for the structure with boron atoms incorporated into the seventh layer. In this case the first two bilayers of diamond (111) surface are reconstructed into two graphene layers. When boron atoms are doped deeper beyond the seventh layer, the situation is instead bulk incorporation and has little effect on the surface.

The relative energies of the boron-doped structures optimized above are summarized in Fig. 3, represented by blue stars. The energies decrease with doping depth in a zigzag way till to the 7 th layer, and then abruptly jump from the 8th layer. Generally, the more the $\mathrm{sp}^{3}$-to-sp ${ }^{2}$ transition happens, the lower the energy is. The energy of the third-layer-doping is lower than the first-layer-doping, because the third-layer-doping causes more $\mathrm{sp}^{3}$-to-sp ${ }^{2}$ transitions and eliminates more surface dangling bonds. The fifth-layer-doping (seventh-layer-doping) has even lower energy, because it has one layer (two layers) of graphene instead of one bilayer (two bi-layers) of diamond compared with the third-layer-doping. The second-, fourth- and sixth-layer-doping have higher energies because these structures reduce the surface dangling bonds less efficiently.

One can see that upon B doping, all relaxed $1 \times 1$ diamond (111) surface energies decrease, due to the elimination of dangling bonds. However, those $1 \times 1$ surfaces can be reconstructed further to 
Pandey-chain structures [39]. The relative energies for Pandey-chain reconstruction surfaces are calculated and represented by black balls in Fig. 3, showing that the energies steadily increase with doping depth starting from the lowest energy in the first layer, and finally approaching the value of the Pandey-chain structure of bulk doping. This indicates that the doping effect on the surface is weaker as the doping boron atom is deeper in Pandey-chain structures, as expected. Now we compare the energies between optimized $1 \times 1$ surface and Pandey-chain surface. In most cases, Pandey-chain surface has lower energy which means that $1 \times 1$ surface can be further reconstructed to Pandey-chain structure. However, when boron is in the fifth (seventh) layer, a dramatic change occurs: the crossover between the blue curve and black curve takes place, whereupon the Pandey-chain structure has higher energy. This clearly shows that the spontaneously formed graphene on diamond (111), if triggered by boron incorporation in the fifth-layer, is the ground state rather than the reconstructed Pandey-chain. As the $\mathrm{sp}^{2}$ hybridized bond is lower in energy than the $\mathrm{sp}^{3}$ bonds, such a reconstruction process favors surface stability more strongly [40]. Afterward, if the substrate below the graphene layer in Fig. 2(d) is further reconstructed into the Pandey-chain structure, the total energy would be much decreased, as denoted by the red square in Fig. 3.

Why does this structure phase transition from diamond $\mathrm{sp}^{3}$ to graphene $\mathrm{sp}^{2}$ happen on diamond (111) surface when boron is doped at the fifth layer, and why is $1 / 4$ doping density enough to make the transition? To understand its origin, we investigated a typical model with a single B atom in a $3 \times 3$ $\underline{\text { surface cell incorporated into the fifth layer of diamond (111) (1/16 boron doping concentration), as }}$ shown in Fig. 2(e). After optimization, a hexagonal graphene-like structure with a bond length of 1.44 A bulges dramatically in the first bilayer, shown in blue in Fig. 2(f). The distance between the three C atoms in purple at the 3rd layer and the three $\mathrm{C}$ atoms at the 2 nd layer, in blue, is elongated to $2.42 \AA$, indicating much weaker bonding, or no more bonding at all, between them [41]. Now, we present in detail a physical description of how a B atom induces formation of the graphene-like ring. Once a boron atom is incorporated into the 5th layer, the B atom forms covalent bonds with its three nearest $\mathrm{C}$ atoms at the 6th layer, while it creates a dangling bond on a $\mathrm{C}_{\text {top }}$ atom at the 4 th layer right on top of the $\mathrm{B}$ atom. In fact, the role of the doped $\mathrm{B}$ atom at the 5th layer is simply to introduce a dangling bond underneath the $\mathrm{C}_{\text {top }}$ atom. Both the $\mathrm{C}_{\text {top }}$ atom and all $\mathrm{C}$ atoms at the surface with dangling bonds have a strong tendency of changing from the $\mathrm{sp}^{3}$ state to the $\mathrm{sp}^{2}$ state in order to reduce the number of dangling bonds. Then, the $\mathrm{C}_{\text {top }}$ atom will negotiate with the three neighboring carbon atoms at the 3rd layer, 
shown in purple in Fig. 2(e), to change together to the $\mathrm{sp}^{2}$ state, and surface atoms persuade $\mathrm{C}$ atoms at the 2nd layer to do the same. Then, those $\mathrm{C}$ atoms at the 2nd layer and 3rd layer will communicate to accept the proposal to be in the $\mathrm{sp}^{2}$ state. Finally, one can see that the $\mathrm{C}_{\mathrm{top}}$ is lifted up to an elongated distance of $1.89 \AA$ and forms $\mathrm{sp}^{2}$ hybridized bonds with the three neighboring carbon atoms, shown in purple in Fig. 2(f), and the six $\mathrm{C}$ atoms on top of the $\mathrm{B}$ in the first bilayer form a $\mathrm{sp}^{2}$ hexagonal graphene-like structure. Thereupon, the three bonds between purple carbon atoms in the 2nd bilayer and the blue atoms in the 1 st bilayer vanish, since all of them have only $\mathrm{sp}^{2}$ hybridized bonds. It is worth emphasizing again that the three $\mathrm{sp}^{3}$ bonds are not broken, but rather disappear due to the transition from $\mathrm{sp}^{3}$ to $\mathrm{sp}^{2}$ state.

So, we have shown that a single doped boron atom at the fifth layer induces formation of one graphene-like ring on the surface. By increasing the doping concentration to $1 / 4$, the first bilayer is completely graphitized as shown in Fig. 2(d). This density requires that each doped B atom must induce the transformation of four carbon $\mathrm{sp}^{3}$ bonds between the 1 st bilayer and the 2 nd bilayer into the $\mathrm{sp}^{2}$ state. Two different processes are responsible for this phase transition, in direct and indirect manners, respectively. The direct process was already described above: a single B atom at the 5th layer creates a six-fold graphite-like ring, where three $\mathrm{sp}^{3}$ bonds have changed to $\mathrm{sp}^{2}$, induced by the $\mathrm{B}$ atom. Now, one more bond is left to be understood.

We need to look again at the six-fold graphite-like ring structure created by a single B atom. Once it is formed, all six $\mathrm{C}$ atoms are in $\mathrm{sp}^{2}$ states, in which three $\mathrm{C}$ atoms at the 2nd layer change to the $\mathrm{sp}^{2}$ state, and three $\mathrm{C}$ atoms at the $1 \mathrm{st}$ layer transform to the $\mathrm{sp}^{2}$ state. Note that the three $\mathrm{C}$ atoms at the $1 \mathrm{st}$ layer will affect neighboring $\mathrm{C}$ atoms at the 2nd layer, outside of the ring. When those graphite-like rings form a periodic structure in our $2 \times 2$ surface cell with $1 / 4 \mathrm{~B}$ doping density, the last $\mathrm{sp}^{3}$-bonded $\mathrm{C}$ atom at the 2 nd layer is surrounded by three graphite-like rings and it connects to three first-layer C atoms in these rings. Together, these three surrounding $\mathrm{sp}^{2}$-state $\mathrm{C}$ atoms enable the last $\mathrm{sp}^{3}$-to-sp ${ }^{2}$ transition of a $\mathrm{C}$ atom at the 2 nd layer. In order to check this picture, we repeated the calculation, eliminating several intermediate steps during the optimization, showing clearly that the graphene rings appear very quickly after just a few optimization steps in which three $\mathrm{sp}^{3}$ bonds atop each $\mathrm{B}$ atom disappear, and later, the B atom's only remaining $\mathrm{sp}^{3}$ bond becomes weaker and weaker, and finally disappear too. This is an indirect process and could be regarded as a 2nd-order effect of doped B atoms, since it results from the collective efforts of three surrounded $\mathrm{C}$ atoms in three rings that formed earlier 
in the process. Eventually, both the direct and indirect processes transform all $\mathrm{C}$ atoms at the 1st bilayer into the $\mathrm{sp}^{2}$ state whereupon the graphene has been automatically constructed.

Finally, we plotted the charge distribution, shown in Fig. 4, for 1/4 concentration of boron incorporated into the fifth layer in diamond (111) [as shown in Fig. 2(d)]. In order to see the differences between the boron-to- $\mathrm{C}_{\text {top }}$ bonding and the bonding of the boron atom to the $\mathrm{C}$ atoms below it, we chose to investigate more closely the plane formed by a boron atom, its $\mathrm{C}_{\text {top }}$ atom and one $\mathrm{C}$ atom below the boron. One can clearly see that boron's bonding to the $\mathrm{C}_{\text {top }}$ atom is much weaker than its bonding to the $\mathrm{C}$ atom below, indicating that the $\mathrm{C}_{\text {top }}$ atom has a dangling bond. As a result, the carbon atoms in the fourth layer shift upward a bit and use their dangling bonds to form stronger $\mathrm{sp}^{2}$ bonds with carbon in the third layer.

\section{Conclusions}

In summary, we investigated the formation of graphene on boron-doped diamond (111) surface by using first-principles calculations, demonstrating that graphene can be spontaneously constructed with $1 / 4$ substitutional doping of boron atoms in the fifth layer of diamond (111). Surface reconstruction induced by the B-doping is the physical origin of the observed diamond-to-graphene phase transition. The 1st bi-layer of diamond (111) becomes graphene due to surface reconstruction, driven by a strong tendency of surface carbon atoms to transform to the $\mathrm{sp}^{2}$ state, combined with the influence of sub-surface doping with B atoms. This result paves the way to a direct approach for graphene synthesis on wide bandgap materials, which will attract significant interest in view of the widespread quest for graphene-based electronics with high thermal conductivity.

\section{Acknowledgments}

This work is supported by the National Natural Science Foundation of China under Grant Nos. 51272278, 61390503, 91323304, and 11574369; MOST under Grant No.2016YFA0200402, XDB07020200, Nanosciences Foundation in Grenoble and French ANR PNANO Project "Nanosim-Graphene," and the US Department of Energy under Grant No. DE-SC0002623. 
References

[1] K.S. Novoselov, A.K. Geim, S.V. Morozov, D. Jiang, Y. Zhang, S.V. Dubonos, I.V. Grigorieva, A.A. Firsov, Electric Field Effect in Atomically Thin Carbon Films, Science 306(2004) 666-669.

[2] K.S. Novoselov, D. Jiang, F. Schedin, T.J. Booth, V.V. Khotkevich, S.V. Morozov, A.K. Geim, Two-dimensional atomic crystals, Proc. Natl. Acad. Sci. 102(2005) 10451-10453.

[3] Y. Zhang, J.W. Tan, H.L. Stormer, P. Kim, Experimental observation of the quantum Hall effect and Berry's phase in graphene, Nature 438(2005) 201-204.

[4] M.F. Yu, O. Lourie, K. Moloni, T.F. Kelly, R.S. Ruoff, Strength and Breaking Mechanism of Multiwalled Carbon Nanotubes Under Tensile Load, Science 287(2000) 637-640.

[5] Y. Zhang, J.P. Small, M.E.S. Amori, P. Kim, Electric Field Modulation of Galvanomagnetic Properties of Mesoscopic Graphite, Phys. Rev. Lett. 94(2005) 176803.

[6] V.V. Cheianov, V. Falko, B.L. Altshuler, The Focusing of Electron Flow and a Veselago Lens in Graphene $p$-n Junctions, Science 315(2007) 1252-1255.

[7] J.R. Williams, L. Dicarlo, C.M. Marcus, Quantum Hall Effect in a Gate-Controlled $p$ - $n$ Junction of Graphene, Science 317(2007) 638-641.

[8] D.A. Abanin, L.S. Levitov, Quantized Transport in Graphene $p$ - $n$ Junctions in a Magnetic Field, Science 317 (2007) 641-643.

[9] C. Berger, Z. Song, T. Li, X. Li, A.Y. Ogbazghi, R. Feng, Z. Dai, A.N. Marchenkov, E.H. Conrad, P.N. First, W.A. de Heer, Ultrathin Epitaxial Graphite: 2D Electron Gas Properties and a Route toward Graphene-based Nanoelectronics, J. Phys. Chem. B 108(2004) 19912-19916.

[10] C. Berger, Z. Song, X. Li, X. Wu, N. Brown, C. Naud, D. Mayou,T. Li, J. Hass, A.N.

Marchenkov, E.H. Conrad, P.N. First, W.A. de Heer, Electronic Confinement and Coherence in Patterned Epitaxial Graphene, Science 312(2006) 1191-1196.

[11] A.K. Geim, K.S. Novoselov, The rise of graphene, Nat. Mater. 6(2007) 183-191.

[12] Y.-M. Son, M.L. Cohen, S.G. Louie, Half-metallic graphene nanoribbons, Nature 444(2006) 347-349.

[13] B. Trauzettel, D.V. Bulaev, D. Loss, G. Burkard, Spin qubits in graphene quantum dots, Nature Phys. 3(2007) 192-196.

[14] T. Yokoyama, Controllable spin transport in ferromagnetic graphene junctions, Phys. Rev. B 77(2008) 073413. 
[15] H. Yang, M. Chshiev, X. Waintal, S. Roche, Proximity Effects Induced in Graphene by Magnetic Insulators: First-Principles Calculations on Spin Filtering and Exchange-Splitting Gaps, Phys. Rev. Lett. 110(2013) 046603

[16] V. I. Falko, Graphene: Quantum information on chicken wire, Nature Phys. 3(2007) 151-152.

[17] K.V. Emtsev, A. Bostwick, K. Horn, J. Jobst, G.L. Kellogg, L. Ley, J.L. McChesney, T. Ohta, S.A. Reshanov, J. Röhrl, E. Rotenberg, A.K. Schmid, D. Waldmann, H. B. Weber, T. Seyller, Towards wafer-size graphene layers by atmospheric pressure graphitization of silicon carbide, Nat Mater. 8(2009) 203-207.

[18] S.Y. Zhou, G.-H. Gweon, A.V. Fedorov, P.N. First, W.A. De Heer, D.-H. Lee, F. Guinea, A.H. Castro Neto, A. Lanzara, Substrate-induced bandgap opening in epitaxial graphene, Nat. Mater. 6(2007) $770-775$

[19] T. Ohta, A. Bostwick, T. Seyller, K. Horn, E. Rotenberg, Controlling the electronic structure of bilayer graphene, Science 313(2006) 951-4.

[20] X.Y. Peng, R. Ahuja, Symmetry Breaking Induced Bandgap in Epitaxial Graphene Layers on SiC, Nano Lett. 8(2008) 4464-4468.

[21] W.A. de Heer, C. Berger, X. Wu, P.N. First, E.H. Conrad, X. Li, T. Li, M. Sprinkle, J. Hass, M.L. Sadowski, M. Potemski, G. Martinez, Epitaxial graphene, Solid State Commun. 143(2007) 92-100.

[22] A.H. Castro Neto, F. Guinea, N.M.R. Peres, K.S. Novoselov, A.K. Geim, The electronic properties of graphene, Rev. Mod. Phys. 81(2009) 109-162.

[23] D.M. Wang, G.R. Chen, C.K. Li, M. Cheng, W. Yang, S. Wu, G.B Xie, J. Zhang, J. Zhao, X.B. Lu, P. Chen, G.L Wang, J.L Meng, J. Tang, R. Yang, C.L He, D.H Liu, D.X Shi, K. Watanabe, T. Taniguchi, J. Feng, Y.B Zhang, G.Y. Zhang, Thermally Induced Graphene Rotation on Hexagonal Boron Nitride, Phys. Rev. Lett. 116(2016) 126101

[24] J. Yu, G. Liu, A.V. Sumant, V. Goyal, A.A. Balandin, Graphene-on-Diamond Devices with Increased Current-Carrying Capacity: Carbon sp ${ }^{2}$-on-sp ${ }^{3}$ Technology, Nano Lett. 12(2012) 1603-1608. [25] Y. Wu, Y. Liu, A.A. Bol, K.A. Jenkins, F. Xia, D.B. Farmer, Y. Zhu, P. Avouris, High-frequency, scaled graphene transistors on diamond-like carbon, Nature 472(2011) 74-78.

[26] F. Zhao, A. Vrajitoarea, Q. Jiang, X.Y. Han, A. Chaudhary, J.O. Welch, R.B. Jackman, Graphene-Nanodiamond Heterostructures and their application to High Current Devices, Scientific Report 5(2015) 13771 
[27] K. Ueda, S. Aichi, H. Asano, Direct formation of graphene layers on diamond by high-temperature annealing with a Cu catalyst, Diamond \& Related Materials 63 (2016) 148-152 [28] K.J. Sankaran, T.H. Chang, S.K. Bikkarolla, S.S. Roy, P. Papakonstantinou, S. Drijkoningen, P. Pobedinskas, M.K. Van Bael, N.H Tai, I.N. Lin, K. Haenen, Growth, structural and plasma illumination properties of nanocrystalline diamond-decorated graphene nanoflakes, RSC Adv. $\underline{6(2016) 63178-63184}$

[29] C.Z. Gu, W.X. Li, J. Xu, S.C. Xu, C. Lu, L.F. Xu, J.J. Li, S.B. Zhang, Graphene grown out of diamond, Appl. Phys. Lett. 109(2016) 162105.

[30] L. Magaud, F. Hiebel, F. Varchon, P. Mallet, J.-Y.Veuillen, Graphene on the C-terminated SiC (0001) surface: An ab initiostudy, Phys. Rev. B 79(2009) 161405(R).

[31] K.S. Novoselov, A. Mishchenko, A. Carvalho, A.H. Castro Neto, 2D materials and van der Waals heterostructures, Science 353(2016)aac9439

[32] V.L. Kuznetsov, I.L. Zilberberg, Y.V. Butenko, A.L. Chuvilin, Theoretical study of the formation of closed curved graphite-like structures during annealing of diamond surface, J. Appl. Phys. 86(1999) 863-870.

[33] Y. Yan, S.B. Zhang, M.M. Al-Jassim, Graphite-like surface reconstructions on C $\{111\}$ and their implication for $n$-type diamond, Phys. Rev. B 66(2002) 201401(R).

[34] G. Kresse, J. Furthmuller, Efficient iterative schemes for $a b$ initio total-energy calculations using a plane-wave basis set, Phys. Rev. B 54(1996) 11169-11186.

[35] D. Vanderbilt, Soft self-consistent pseudopotentials in a generalized eigenvalue formalism, Phys. Rev. B 41(1990) 7892-7895.

[36] Y.Wang, J.P. Perdew, Correlation hole of the spin-polarized electron gas, with exact small-wave-vector and high-density scaling, Phys. Rev. B 44(1991) 13298-13307

[37] H.J. Monkhorst, J.D. Pack, Special points for Brillouin-zone integrations, Phys. Rev. B 13(1976) 5188-5192.

[38] K.C. Pandey, New dimerized-chain model for the reconstruction of the diamond $(111)-(2 \times 1)$ surface, Phys. Rev. B 25(1982) 4338-4341.

[39] S. Samlenski, C. Haug, R. Brenn, C. Wild, R. Locher, P. Koidl, Characterisation and lattice location of nitrogen and boron in homoepitaxial CVD diamond, Diam. Relat. Mater. 5(1996) 947-951. [40] A.S. Barnard, M. Sternberg, Substitutional Boron in Nanodiamond, Bucky-Diamond, and 
Nanocrystalline Diamond Grain Boundaries, J. Phys. Chem. B 110(2006) 19307-19314.

[41] S.N. Zhao, K. Larsson, First principle study of the attachment of graphene onto non-doped and doped diamond (111), Diamond and Related Materials 66(2016)52-60 


\section{Figure captions}

FIG. 1: (a) Side view of diamond (111). (b) Top view of 1 bilayer of diamond (111); big gray balls represent carbon atoms in the first layer, while small blue balls indicate carbon atoms in the second layer.

FIG. 2: (a) and (b) Optimized diamond (111) with $1 / 4$ of the carbon atoms in the first and third layer replaced by boron atoms, respectively. (c) and (d) Initial and optimized structures, respectively, both with 1/4 of the carbon atoms in the fifth layer replaced by boron atoms. (e) and (f) Initial and optimized structures, respectively, both with 1/16 boron doping concentration in the fifth layer. Pink balls represent incorporated boron atoms, and grey balls indicate carbon atoms. In (e) and (f), the blue balls represent carbon atoms before and after forming hexagonal graphite structure in the first bilayer, while the purple balls represent carbon atoms before and after forming $\mathrm{sp}^{2}$ bonds with $\mathrm{C}_{\text {top }}$ atom.

FIG. 3: Relative energy of 1/4 boron doped diamond (111) with $1 \times 1$ reconstructed and Pandey-chain reconstructed surfaces as functions of the depth of boron incorporation. Lines serve only to guide the eye.

FIG. 4: Charge distribution (e/ $\AA^{2}$ ) across the plane determined by boron (white), the nearest carbon (black) on top of boron and one of the nearest carbon below boron for the 1/4 boron incorporated in fifth layer diamond of Fig. 2(d). A key to the other colors is shown on the right side - the gradient from blue to red shows charge density from lower to higher. 

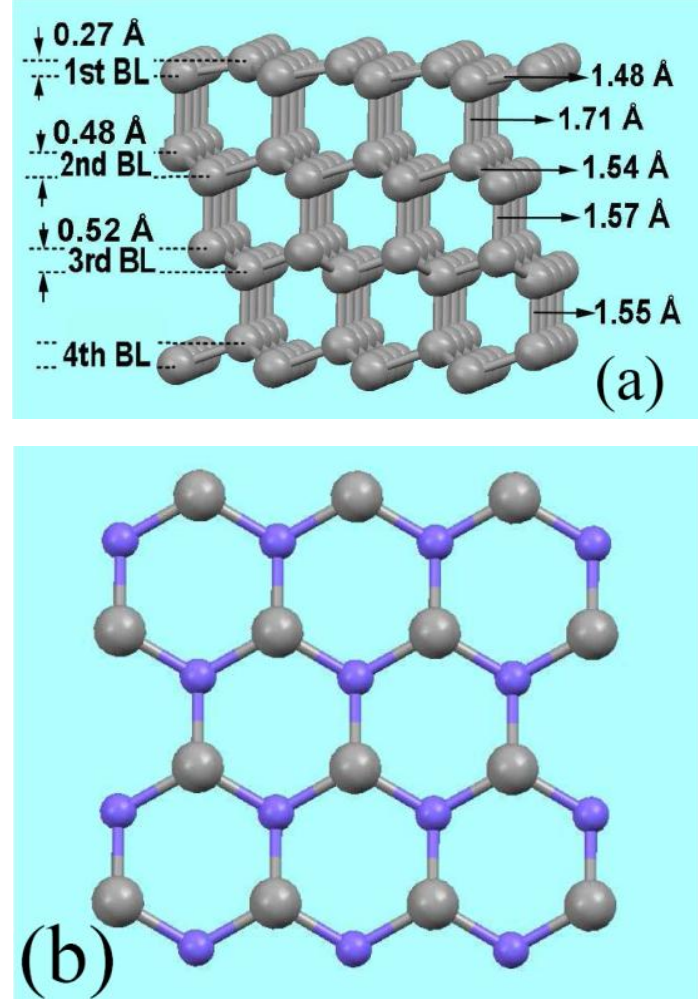

FIG. 1: (a) Side view of diamond (111). (b) Top view of 1 bilayer of diamond (111); big gray balls represent carbon atoms in the first layer, while small blue balls indicate carbon atoms in the second layer. 

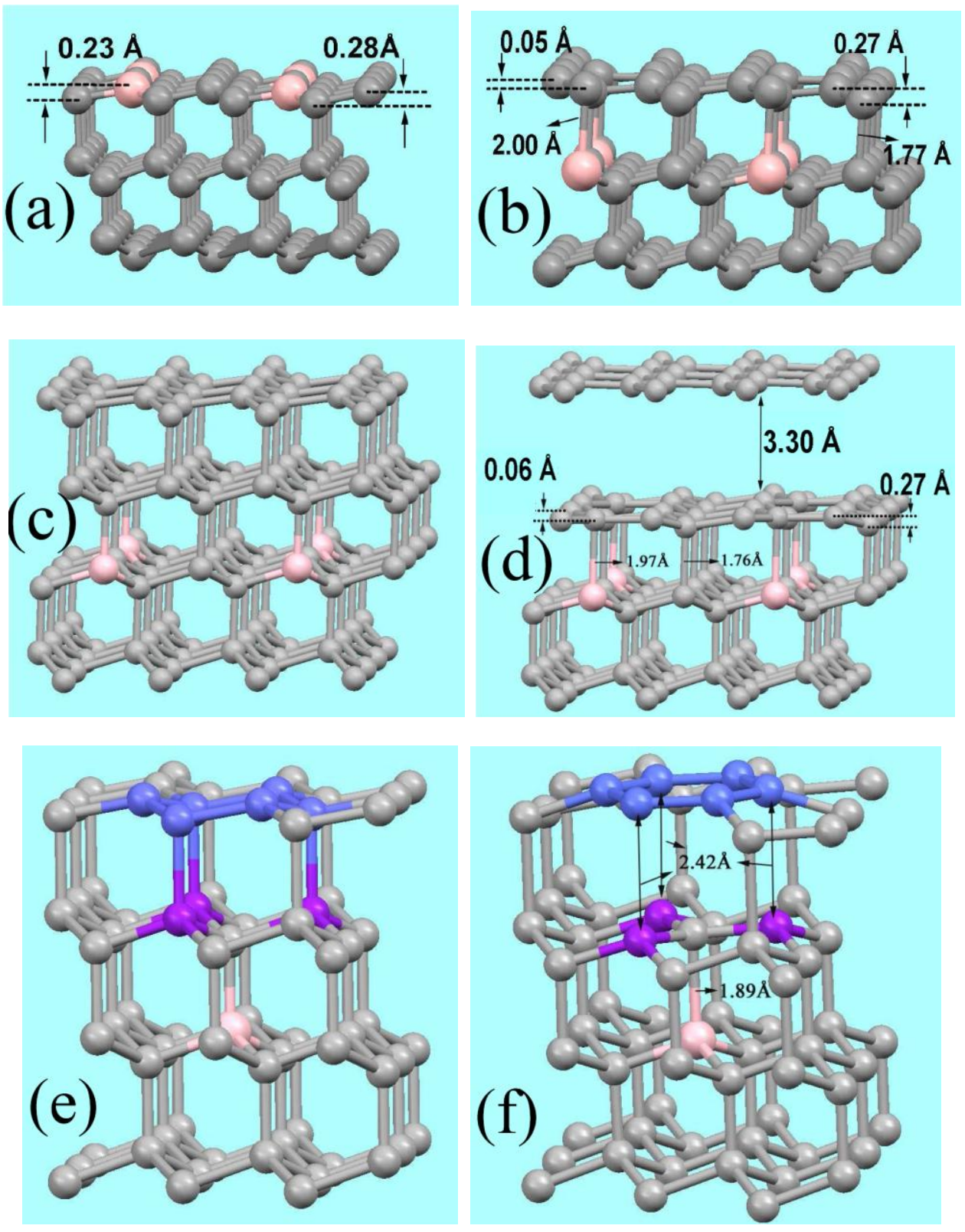

FIG. 2: (a) and (b) Optimized diamond (111) with $1 / 4$ of the carbon atoms in the first and third layer replaced by boron atoms, respectively. (c) and (d) Initial and optimized structures, respectively, both with 1/4 of the carbon atoms in the fifth layer replaced by boron atoms. (e) and (f) Initial and optimized structures, respectively, both with 1/16 boron doping concentration in the fifth layer. Pink balls represent incorporated boron atoms, and grey balls indicate carbon atoms. In (e) and (f), the blue balls represent carbon atoms before and after forming hexagonal graphite structure in the first bilayer, while the purple balls represent carbon atoms before and after forming $\mathrm{sp}^{2}$ bonds with $\mathrm{C}_{\mathrm{top}}$ atom. 


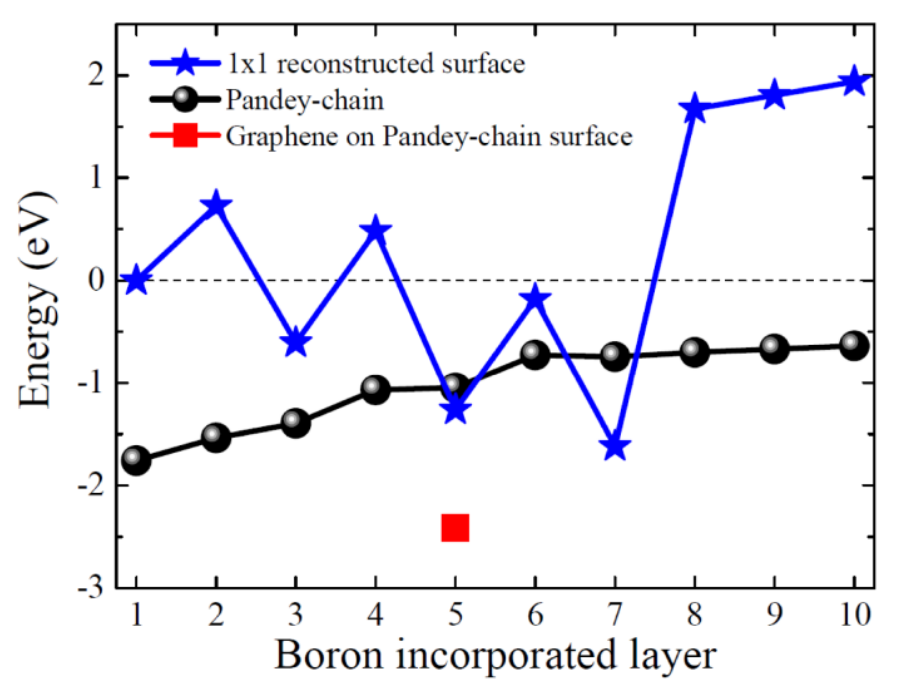

FIG. 3: Relative energy of $1 / 4$ boron doped diamond (111) with $1 \times 1$ reconstructed and Pandey-chain reconstructed surfaces as functions of the depth of boron incorporation. Lines serve only to guide the eye. 


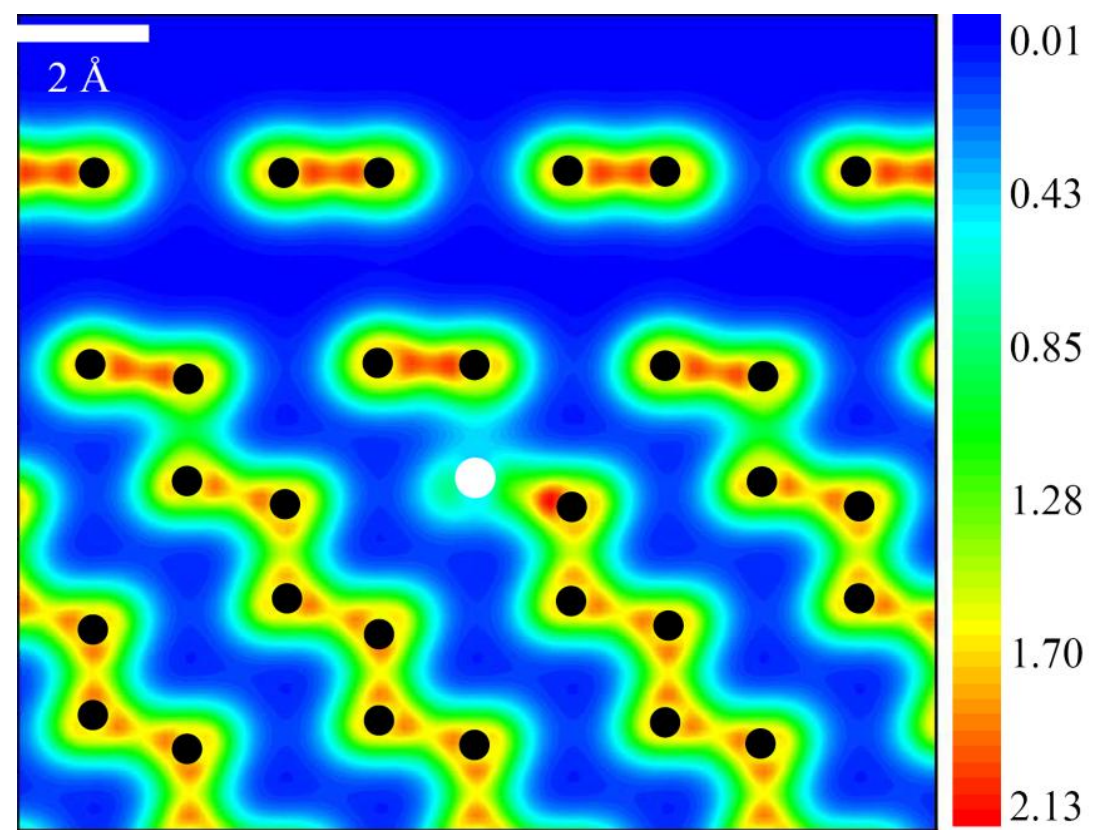

FIG. 4: Charge distribution (e/ $\AA^{2}$ ) across the plane determined by boron (white), the nearest carbon (black) on top of boron and one of the nearest carbon below boron for the $1 / 4$ boron incorporated in fifth layer diamond of Fig. 2(d). A key to the other colors is shown on the right side - the gradient from blue to red shows charge density from lower to higher. 


\section{Graphical abstract}

Spontaneous construction of graphene on boron-doped diamond (111) surface has been found in first-principles calculations. Boron-doping-induced surface reconstruction is the mechanism responsible for the diamond-to-graphene phase transition. Furthermore, the effect on the surface is unexpectedly observed at any doping depth down to the 7th layer, and a low concentration of substituent boron - only $1 / 4$ - is effective for graphene formation. Amazingly, when boron atoms are incorporated into the fifth layer, the direct optimization of the $1 \times 1$ surface automatically gives rise to a graphene structure from the first bilayer with no energy barrier, indicating that the formation of graphene is spontaneous. These findings provide an alternative strategy for graphene synthesis on wide-gap insulators with high thermal conductivity.
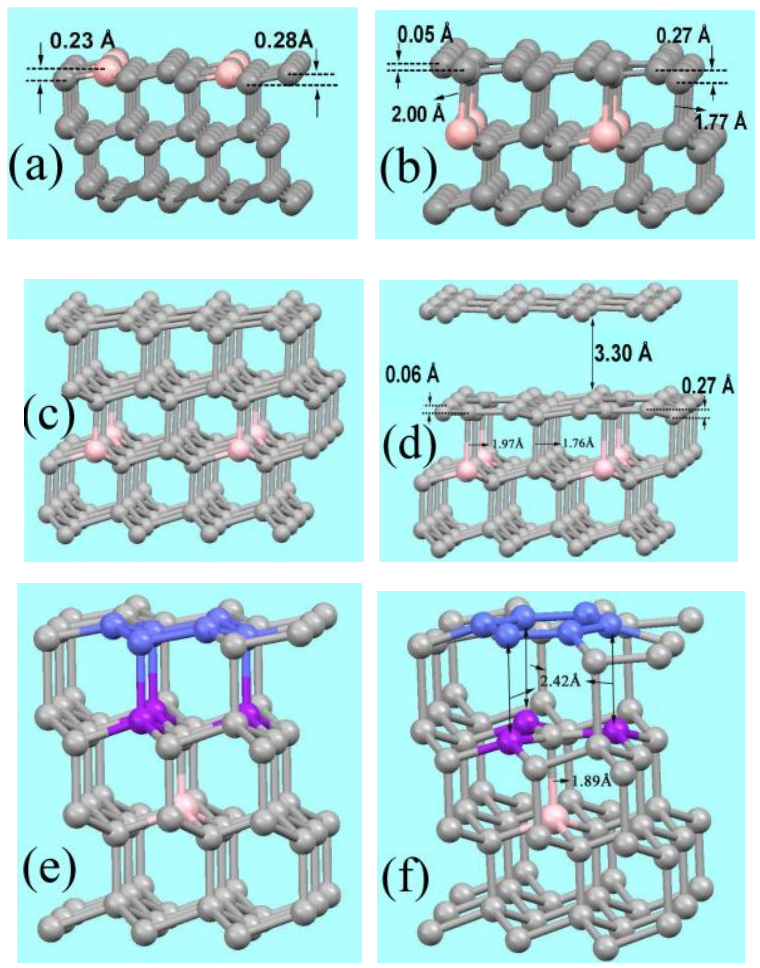

(a) and (b) Optimized diamond (111) with 1/4 of the carbon atoms in the first and third layer replaced by boron atoms, respectively. (c) and (d) Initial and optimized structures, respectively, both with 1/4 of the carbon atoms in the fifth layer replaced by boron atoms. (e) and (f) Initial and optimized structures, respectively, both with 1/16 boron doping concentration in the fifth layer. Pink balls represent incorporated boron atoms, and grey balls indicate carbon atoms. In (e) and (f), the blue balls represent carbon atoms before and after forming hexagonal graphite structure in the first bilayer, while the purple balls represent carbon atoms before and after forming $\mathrm{sp}^{2}$ bonds with $\mathrm{C}_{\text {top }}$ atom. 